\title{
Inhibition-Based Biomarkers for Autism Spectrum Disorder
}

\author{
April R. Levin $^{1,2}$ - Charles A. Nelson ${ }^{2,3}$
}

Published online: 27 March 2015

(C) The American Society for Experimental NeuroTherapeutics, Inc. 2015

\begin{abstract}
Autism spectrum disorder (ASD) is a behaviorally defined and heterogeneous disorder. Biomarkers for ASD offer the opportunity to improve prediction, diagnosis, stratification by severity and subtype, monitoring over time and in response to interventions, and overall understanding of the underlying biology of this disorder. A variety of potential biomarkers, from the level of genes and proteins to network-level interactions, is currently being examined. Many of these biomarkers relate to inhibition, which is of particular interest because in many cases ASD is thought to be a disorder of imbalance between excitation and inhibition. Abnormalities in inhibition at the cellular level lead to emergent properties in networks of neurons. These properties take into account a more complete genetic and cellular background than findings at the level of individual genes or cells, and are able to be measured in live humans, offering additional potential as diagnostic biomarkers and predictors of behaviors. In this review we provide examples of how altered inhibition may inform the search for ASD biomarkers at multiple levels, from genes to cells to networks.
\end{abstract}

April R. Levin

april.levin@childrens.harvard.edu

1 Department of Neurology, Boston Children's Hospital/Harvard Medical School, Boston, MA, USA

2 Laboratories of Cognitive Neuroscience, Boston Children's Hospital/ Harvard Medical School, Boston, MA, USA

3 Harvard Graduate School of Education, Cambridge, MA, USA
Keywords Autism spectrum disorder (ASD) · Biomarkers · Inhibition $\cdot \mathrm{GABA} \cdot$ Interneurons $\cdot$ Networks

\section{Background}

The majority of neurodevelopmental disorders are behaviorally defined [1]. The search for biomarkers in these disorders is thus enticing as they offer the opportunity to understand and diagnose these disorders at a neurobiological level, improving our understanding of their underlying mechanisms and thus moving neurodevelopmental disorders from the realm of behavior and mind to biology and brain. Additionally, biomarkers offer the opportunity to predict, diagnose, stratify, and monitor diseases over time and in response to intervention. However, the search for biomarkers has historically been driven and complicated by heterogeneity among neurodevelopmental disorders [2]; that is, the tremendous overlap in signs and symptoms among disorders and the comorbid conditions that co-exist within any given disorder [3].

Autism spectrum disorder (ASD) is one such disorder. In the USA, ASD affects approximately 1 in 68 children (1:42 males) [4]; it is also highly heterogeneous. While core behavioral symptoms of difficulties with social communication and restricted/repetitive behaviors are necessary to make the diagnosis [1], the spectrum of difficulty in each of these areas is wide. Further heterogeneity is brought about by the vast array of comorbidities (e.g., those related to sleep, epilepsy, motor function, gastrointestinal, immune, and other neurologic and psychiatric comorbidities) with which each individual child with ASD may or may not present [5]. Additionally, the search for biomarkers of autism has been equally marked by heterogeneity, with proposed biomarkers including findings as diverse as genetic variants; gene expression; biochemical markers; immune responses; morphological markers such as 
head size and dysmorphic features; neuroanatomical findings [e.g., on magnetic resonance imaging (MRI)]; electrophysiological findings [e.g., on electroencephalography (EEG)], and neuropsychological and behavioral findings [6-8]. Despite this wide array of potential biomarkers, correlation between these findings and the clinical diagnosis of ASD has remained elusive. For example, while numerous genetic disorders drastically increase the risk of ASD, there is no known genetic disorder in which the penetrance of ASD is $100 \%$. A genetic mutation known to be associated with autism may result in typical development, or alternative disorders such as language impairment, intellectual disability, epilepsy, or schizophrenia [9].

Autism is frequently hypothesized to be a disorder of imbalance between excitation and inhibition [10]. While such an imbalance may also contribute to a number of other neurodevelopmental and psychiatric disorders [11], this hypothesis provides the basis for a number of ASD biomarkers at various levels, from molecules to the neural networks that ultimately determine behavior. Therefore, while several prior reviews have focused on various types of biomarkers in ASD $[5-8,12,13]$, here we focus specifically on potential biomarkers associated with altered inhibition, using them as an example of the types of biomarkers that will ultimately be of benefit in ASD. This paper therefore has several goals: 1) we will begin by discussing the types of biomarkers being proposed in ASD, and how these biomarkers may benefit ASD diagnosis and management; 2) next, we will outline current understanding of inhibition in ASD at multiple levels, from cells to networks to behavior, and describe ways in which this understanding augments the hypothesis-driven search for ASD biomarkers - this section includes several examples of how inhibition is altered in ASD, at the level of a) cells and b) networks; 3) finally, we will end with a discussion of future directions in the field of inhibition-based ASD biomarkers.

\section{Types of Biomarkers}

A biomarker is defined by the Biomarkers Definitions Working Group [14] as "A characteristic that is objectively measured and evaluated as an indicator of normal biological processes, pathogenic processes, or pharmacologic responses to a therapeutic intervention". Biomarkers have a number of potential applications, including identifying patients with a disorder, stratifying severity and disease subtypes, indicating prognosis, and predicting and monitoring clinical response to an intervention. For neurodevelopmental disorders, they also augment the biological understanding of an otherwise behaviorally defined disorder. Some (but not all) biomarkers can be used as surrogate end points in clinical trials; to be used in this manner, they must be likely to predict clinical benefit [14].

Evaluation of a biomarker as a surrogate end point is an ongoing process. Biomarkers must be proven to be able to successfully and reproducibly measure an objective, quantifiable clinical characteristic (measurement validity). They must also correlate strongly with the clinical end point of interest in a given population and situation (internal validity). This correlation must then also extend to other populations and situations (external validity), with ongoing evaluation necessary to determine the populations, treatments, and clinical end points for which the biomarker provides a legitimate surrogate end point [15].

In ASD, research has also focused on identifying markers that indicate high risk for ASD, with the eventual goal of developing early specific predictive biomarkers $[7,12]$. The motivation for this research has stemmed from the idea that early initiation of intensive therapy for ASD leads to significantly improved outcomes [16], but early therapy requires early diagnosis. Diagnosis by behavioral measures has been validated only in children as young as 12-21 months [17, 18], and then only in specialized clinical programs. The average age of diagnosis in the general community frequently occurs later, at an average of $4-6$ years of age $[19,20]$, postponing the initiation of much-needed aggressive intervention in these children. Identifying early biomarkers that predict autism would therefore allow for earlier initiation of treatment, with the goal of improving outcomes [21].

\section{Inhibition in ASD: From Molecules to Networks to Behavior}

Autism is frequently hypothesized to be a disorder of imbalance between excitation and inhibition [10]. This does not necessarily mean a type of linearity among excitation and inhibition, as if the brain is a balance scale that can be tipped toward excitation or inhibition. Instead, a key aspect of inhibition lies in its ability to generate complexity and nonlinearity in brain activity. Imagine, for example, a chain of 3 inhibitory neurons. The first neuron may suppress the activity of the second neuron, which will, in fact, result in an increase of activity in the third neuron [22]. Thus, it quickly becomes clear that excessive or impaired inhibition can have complex, unexpected, and difficult to predict downstream effects. However, in the case of ASD, such effects may provide a useful framework for thinking about the diverse and seemingly contradictory clinical findings often seen in ASD.

Neuronal connectivity is also believed to be altered in ASD [23-26]. This hypothesis may be complementary to hypotheses about an imbalance in excitation/inhibition. Establishing, tuning, and maintaining appropriate connectivity in the brain requires a series of steps, each of which may lead to (or be affected by) altered excitatory/inhibitory balance.

\section{Inhibition at the Molecular and Cellular Level}

Inhibition in the central nervous system is primarily accomplished by interneurons using the neurotransmitter $\gamma$ - 
aminobutyric acid (GABA). Neuronal generation, migration, and differentiation are the first steps in establishing appropriate connectivity [27]. Once cells are in place, synaptic adhesion must then sufficiently anchor the connections between 2 neurons, so that communication can occur [28]. Next, the state of each neuron (and its geographic and functional neighbors within a network) must be continually modulated in order to allow recognition of meaningful synaptic inputs while filtering out spurious input. Of particular interest in GABAergic neurons is the maturational switch of postsynaptic GABAergic responses from depolarizing to hyperpolarizing (i.e., excitatory to inhibitory), which generally occurs during the early postnatal period [27]. Meaningful synaptic inputs must then stimulate activity-dependent transcription and translation, so that groups of neurons have "memory" of a previously meaningful interaction and can respond more robustly to similar interactions in the future [29]. Finally, immune mechanisms must be adequately tuned to maintain and support robust synapses, while pruning synapses that are spurious or weak [30].

Abnormalities at any of these steps can lead to altered inhibition. Several such abnormalities merit further exploration as potential biomarkers, although their utility for diagnosis in humans (except postmortem) may currently be limited. For example, compared with controls, numerous models of ASD demonstrate decreased numbers of parvalbumin-positive inhibitory interneurons in the brain [31]. However, even when cells are in place, their functional inhibitory activity may be altered. Postmortem neuropathological studies of people with ASD have demonstrated decreased GABA precursors in certain cell populations, and decreased number, density, and expression of GABA receptors in select cortical areas [32]. Notably, other postmortem studies have demonstrated increased numbers of parvalbumin-positive inhibitory interneurons in the hippocampus, raising the possibility that a compensatory decrease in $\mathrm{GABA}_{\mathrm{A}}$ receptors might be occurring there [33].

Methyl CpG binding protein 2 (MeCP2) mutations, which can underpin Rett syndrome, are also associated with ASD [34]. Clinical testing for these mutations is currently available, but the electrophysiological manifestations of such mutations at the level of individual cells is being explored predominantly in animal models. In mice, cortical GABAergic neurons express more MeCP2 than non-GABAergic neurons; loss of MeCP2 from GABAergic neurons (sparing non-GABAergic neurons) leads to reduced GABA precursors presynaptically, decreases the amplitude of mini inhibitory postsynaptic currents, and recapitulates many features of Rett syndrome [35, 36].

Other genetic mutations are associated with altered inhibition at different stages in the development of structural and functional interneuron connectivity. Fragile $\mathrm{X}$ is associated with increased ASD risk in humans [37]. In an FMR1 knockout model of Fragile X, animals demonstrated decreased numbers of parvalbumin-positive inhibitory interneurons [38], region-specific decreases in expression of $\mathrm{GABA}_{\mathrm{A}}$ receptor subunits, and associated impairments in GABA circuitry [39-41]. In mice with the human R415C mutation in Nlgn3, which affects synaptic adhesion, numbers of parvalbuminpositive inhibitory interneurons are again decreased [31], with an associated increase in levels of vesicular transporters for GABA, frequency of spontaneous inhibitory currents, and amplitude of evoked inhibitory currents [36, 42]. As noted above, the nonlinearity of inhibitory function may help to explain why both increases and decreases in inhibitory function can alter homeostasis [43], and lead to similar-appearing effects at the network and behavioral levels.

Inhibition may also affect, and be affected by, immune reactivity in the nervous system. Postmortem gene expression studies have demonstrated that, compared with controls, in addition to genes related to synaptic function, genes related to immune reactivity are overexpressed in brains of individuals with ASD [44]. A subset of children with ASD have plasma antibodies that autoreact with GABAergic neurons in multiple brain regions [45]. In a properly functioning immune system, synapses are pruned in an activity-dependent manner, pruning synapses with low activity levels and maintaining synapses with high activity levels [30]. Altered pruning can thus disrupt synaptic connectivity [46], and abnormalities in glial function have been associated with difficulties with prepulse inhibition, the acoustic startle response, and social behavior in animal models [47].

Viewed in this light, it becomes easier to see how multiple factors could be implicated in ASD. Mild abnormalities at several steps (particularly during the period of rapid brain development that occurs during late prenatal and early postnatal life) could compound to produce abnormal synaptic connectivity and hence behaviors associated with ASD. In other cases, a severe abnormality at one step may be rescued by a compensatory response elsewhere. For example, synaptic protein synthesis is exaggerated in a mouse model of Fragile $\mathrm{X}$ but suppressed in a mouse model of tuberous sclerosis complex; however, in double mutants, these deviations are corrected [48].

Similarly, genetic testing in humans can identify abnormalities, such as those described above, that lead to altered inhibition. However, penetrance of symptomatology in humans with these genetic findings is variable, likely because of compounding and compensation that can occur downstream. As no genetic mutation is known to have $100 \%$ penetrance for ASD, these mutations can act as biomarkers of risk; however, their presence is not diagnostic for ASD and cannot currently be used to measure outcomes over time.

Therefore, to identify diagnostic and outcome-relevant biomarkers, it is necessary to look downstream from these genetic findings. Manifestations of abnormal inhibition at the cellular level are certainly worth considering, given the findings described above. However, further testing is necessary to 
determine the extent to which the specific cellular abnormalities in inhibition seen in animal models also apply in humans, as findings in humans do not always recapitulate those found in animal models. Unfortunately, in many cases, findings in animals at the cellular level cannot be safely recapitulated in live humans using currently available technologies.

The next step, then, is to look at network-level manifestations of altered inhibition. As a potential biomarker, this offers 2 advantages. First, by describing interactions among large groups of neurons, network-level findings take into account a more complete genetic background, and more complete set of single-cell manifestations, than findings at the level of individual genes or synapses. This is of particular importance because behavior comes not just from individual genes and cells, but from the interactions among these components at the network level. Additionally, network-level findings are measurable in live humans, using noninvasive techniques such as EEG, MRI, and magnetoencephalography (MEG).

\section{Inhibition at the Network Level}

The brain is an enormous network of neurons, the interactions of which lead to emergent properties well beyond those seen at the level of the individual neuron or synapse. These emergent properties are what ultimately lead to the clinical and behavioral manifestations of ASD. Inhibitory interneurons play a key role in this regard, synchronizing action potential generation in neuronal subsets and thus allowing for precise temporal integration across networks of neurons [27, 49], affecting size and propagation of neuronal assemblies, and allowing the generation of oscillations at multiple frequencies, including gamma [27, 31]. These "scaled-up" abnormalities are best seen by studying brain function at the network level.

In humans, neuroimaging techniques (e.g., EEG, MEG, MRI) can probe these network-level abnormalities. MRI offers outstanding spatial resolution when localization of processes is of particular interest, whereas EEG and MEG offer outstanding temporal resolution for examining moment-tomoment activity of large groups of neurons when questions about oscillations, neural "noise", and temporal binding arise.

One example of a finding at the cellular level scaled up to the network level (and behavioral level) involves the activity of oxytocin. As described above, modulation of the state of neurons allows them to recognize meaningful inputs while ignoring spurious inputs. Oxytocin is one such neuromodulator. Because oxytocin levels are reduced in ASD [50], oxytocin has been of recent therapeutic interest. From a behavioral standpoint, oxytocin administration may increase the ability of people with ASD to infer others' expression of emotion [51], although the overall benefits of oxytocin in ASD vary depending on the outcome variable in question [52]. At the synaptic level, oxytocin increases spontaneous inhibitory tone, so that excitatory neurons are less likely to be in a refractory period from spontaneous activity when a meaningful signal arrives, and are therefore more likely to transmit that signal reliably [53]. This highlights the importance of inhibition in improving the signal-to-noise ratio of excitatory transmission. It also raises the possibility that in at least some types of ASD, inadequate inhibitory tone could lead to less faithful transmission of relevant stimuli.

How can such a finding be scaled up to the network level, where it would become detectable noninvasively in humans? In one such model [54], MEG functions as a scaled-up version of intracellular recordings, with gamma oscillations (high-frequency activity that is locally phase-locked) measured instead of spikes in response to a stimulus, and intertrial coherence (the fraction of MEG signals that are in-phase across multiple trials of a time-locked activity) used as a marker of fidelity of information transmission. In human and mouse models of ASD $[55,56]$, intertrial coherence is lower than in controls, suggesting that a smaller number of signals are in phase across trials [54]. Evoked gamma power is also lower than in controls [55-57], suggesting a lower amplitude (and thus lower number of involved neurons) of high-frequency activity in response to trials, on average [54].

These findings mimic those seen at the single-cell level prior to oxytocin administration: inadequate baseline inhibitory tone allows excessive spontaneous excitatory activity to occur, which reduces the fidelity in phase to a specific stimulus. Notably, baseline gamma power is higher in an autism model than in controls [54], just as spontaneous excitatory cell firing is higher before oxytocin administration than after [53]. Additional studies are certainly necessary to better understand the exact biological underpinnings of the similarities seen at these 2 levels. However, overall, these MEG findings seem to quite closely mimic those seen at the single-cell level prior to oxytocin administration: inadequate baseline inhibitory tone at baseline allows excessive spontaneous excitatory activity to occur, which reduces the fidelity of information transfer in response to a stimulus.

This leads, then, to one potential category of inhibitionrelated ASD biomarkers: those related to intraindividual response variability. It has long been noted that autism is a disorder marked by heterogeneity among patients, but that is not the issue of interest here. Instead, it is worth considering the extent to which fidelity of information transfer across trials in a given individual may be altered in ASD. Indeed, the study described above used an auditory paradigm; in a visual paradigm, intraindividual alpha band coherence (the degree to which EEG is phase-locked to a specific event), as well as the amplitude and latency of P1 (a positive-going component occurring about $100 \mathrm{~ms}$ after stimulus onset), all suggested increased between-trial variability in individuals with ASD compared with controls [58]. Other studies have examined trial-by-trial evoked functional MRI responses to visual, auditory, and somatosensory stimuli $[59,60]$, as well as responses 
to movement [61], in patients with ASD, and again found that response reliability was significantly weaker in ASD.

In another category of network-level inhibition-related findings in ASD, phase amplitude coupling (PAC; in which the phase of a slow oscillation modulates the amplitude of a fast oscillation) measured by MEG in response to faces has been found to be reduced in people with ASD compared with controls; additionally, the degree of reduction in PAC correlated with the social score on the Autism Diagnostic Observation Schedule, offering potential as a stratification or prognostic biomarker [62]. Interestingly, similar reductions in PAC were found in mice in which GABAergic inhibition onto parvalbumin-positive interneurons was removed, suggesting altered inhibition as a potential underlying mechanism for this finding [63]. This again highlights the complexities of inhibition; in this study, inhibiting an inhibitory interneuron led to reduced PAC, a far more complex and nonlinear downstream effect of altered inhibition than an arithmetic "double negative" might have predicted.

An additional network-level finding related to inhibition in ASD is that of EEG phase resets. On EEG, the oscillations in clusters of neurons are found to be phase-locked for periods of time, alternating with rapid transitions to new phase-locked states [64]; these transitions appear to be mediated by GABA in thalamocortical circuits [65]. In people with autism, phase lock durations in the 10-12-Hz frequency band were longer than in controls [65], suggesting yet another possible diagnostic or predictive biomarker related to inhibition in ASD.

\section{Future Directions}

The search for biomarkers in ASD has taken multiple forms. Findings in humans and animal models at the level of genes, cells, synapses, and networks each offer strengths and weaknesses in terms of their likely contributions to future biomarkers. Genetic findings, while easily measurable in humans, currently tend to be biomarkers for ASD risk rather than ASD diagnosis or outcome. Cellular and synaptic findings, while difficult to evaluate in live humans, have led to numerous treatment targets addressing potential underlying mechanisms of ASD. Finding surrogate or indirect ways to evaluate for these abnormalities could drastically improve our ability to stratify people with ASD into subgroups likely to respond to a given treatment [13]. Network-level findings, which can be evaluated in humans of all ages using noninvasive techniques such as EEG, MEG, and MRI, offer a window into the emergent properties of groups of neurons whose individual properties may combine to exacerbate or compensate for one another. Because these network-level findings are near the end of the pathway from molecules to behavior, networkbased measurements offer opportunities as potential diagnostic or even predictive biomarkers for the behavioral manifestations of ASD and related disorders, and may eventually offer utility as surrogate end points in clinical trials. Ultimately, then, many avenues of research related to ASD may inform a set of biomarkers useful in understanding, predicting, diagnosing, stratifying, treating, and measuring outcomes in ASD. Findings related to inhibition offer one such set of potential biomarkers, but given the heterogeneity inherent in ASD other findings are also likely to offer useful and complementary biomarkers.

It is worth noting that the intermediate steps between cellular findings and network-level findings in autism are still under investigation. Many of the findings seen in animal models have yet to be replicated in humans, and our understanding of the relationships among findings at the genetic, cellular, network, and behavioral levels remains limited. However, this should not necessarily prevent researchers from investigating hypothesis-driven potential biomarkers that may be new or exploratory. A plethora of data has been collected in various studies of ASD; many of these data are now being catalogued using repositories such as the National Database for Autism Research, offering the opportunity for exploratory analyses that use previously collected data to examine a hypothesis by viewing the data from a new angle. EEG is an example of a source for identifying such biomarkers. EEG contains an extraordinary amount of information, which can be analyzed using a myriad of different signal processing algorithms. There is not one "correct" approach for analyzing EEG data; instead, the approach of interest should be chosen depending on the hypothesis being tested. Therefore, once primary standard analyses (e.g., amplitude and latency of specific event-related potential components [66]) are complete for a given study, EEG data remain available for additional hypothesis testing. This requires time spent on analysis but does not necessarily require additional patient participation or new data collection. This opportunity comes with caveats, of course. First, the team doing additional analyses must maintain a thorough grasp of the task, data collection methods, and diagnostic algorithms used in the population whose data they are using; data from a poorly phenotyped sample, or from a task that may confound the particular analysis a researcher has chosen, is not useful. Second, analyses must either be hypothesis driven or maintain adequate statistical rigor if done in an "unbiased" manner; the plethora of data that a single EEG contains otherwise allows for infinite analyses, which can easily lead to spurious conclusions. However, if used responsibly, repositories of EEG and other data offer low-risk opportunities to investigate potential new biomarkers for ASD.

Given the degree of heterogeneity that is currently fundamental to our understanding of ASD, the ongoing search for diagnostic and predictive biomarkers of ASD is likely to be a 2 -way process. The core behavioral symptoms of ASD remain, for now, the "gold standard" diagnostic criteria. No single genetic study, EEG, MRI, or other test has been found 
to be more reliable in diagnosing ASD than clinician report of Diagnostic and Statistical Manual, Fifth Edition, criteria. However, as the search for ASD biomarkers expands at multiple levels, our understanding of the biology underlying ASD is also likely to expand. Over time, the search for biomarkers of ASD offers the opportunity not only to identify diagnostic and predictive findings, but also to change our fundamental understanding of how alterations in the inner workings of the brain lead to specific behaviors.

Acknowledgments This work was supported by the Nancy Lurie Marks Family Foundation, American Brain Foundation, and Autism Science Foundation (A.R.L.); and the National Institutes of Health (DC 010290) and the Simons Foundation (C.A.N.).

Required Author Forms Disclosure forms provided by the authors are available with the online version of this article.

\section{References}

1. American Psychiatric Association. Diagnostic and statistical manual of mental disorders, fifth edition. American Psychiatric Publishing, Arlington, VA, 2013.

2. Clark LA, Watson D, Reynolds S. Diagnosis and classification of psychopathology: Challenges to the current system and future directions. Annu Rev Psychol 1995;46:121-153.

3. Cuthbert BN, Insel TR. Toward the future of psychiatric diagnosis: The seven pillars of RDoC. BMC Med 2013;11:126.

4. CDC. Prevalence of autism spectrum disorder among children aged 8 years - autism and developmental disabilities monitoring network, 11 sites, United States, 2010. Morbidity and mortality weekly report. Surveillance summaries. Centers for Disease Control, Washington, DC, 2014.

5. Veenstra-VanderWeele J, Blakely RD. Networking in autism: Leveraging genetic, biomarker and model system findings in the search for new treatments. Neuropsychopharmacology 2012;37: 196-212.

6. Ruggeri B, Sarkans U, Schumann G, Persico AM. Biomarkers in autism spectrum disorder: The old and the new. Psychopharmacology (Berl) 2014;231:1201-1216.

7. Voineagu I, Yoo HJ. Current progress and challenges in the search for autism biomarkers. Dis Markers 2013;35:55-65.

8. Goldani AAS, Downs SR, Widjaja F, Lawton B, Hendren RL. Biomarkers in autism. Front Psychiatry 2014;5:100.

9. Murdoch JD, State MW. Recent developments in the genetics of autism spectrum disorders. Curr Opin Genet Dev 2013;23:310315.

10. Rubenstein J, Merzenich M. Model of autism: Increased ratio of excitation/inhibition in key neural systems. Genes Brain Behav 2003;2:255-267.

11. Baroncelli L, Braschi C, Spolidoro M, Begenisic T, Maffei L, Sale A. Brain plasticity and disease: A matter of inhibition. Neural Plast 2011;2011:1-11.

12. Pierce K, Liptak GS. The power and promise of identifying autism early: Insights from the search for clinical and biological markers. Ann Clin Psychiatry 2009;21:132-147.

13. Bent $\mathrm{S}$, Hendren RL. Improving the prediction of response to therapy in autism. Neurotherapeutics 2010;7:232-240.

14. Biomarkers Definitions Working Group. Biomarkers and surrogate endpoints: preferred definitions and conceptual framework. Clin Pharmacol Ther 2001;69:89-95.
15. Strimbu K, Tavel JA. What are biomarkers? Curr Opin HIV AIDS 2011;5:463-466.

16. Wallace KS, Rogers SJ. Intervening in infancy: Implications for autism spectrum disorders. J Child Psychol Psychiatry 2010;51: $1300-1320$.

17. Zwaigenbaum L, Bryson S, Rogers T, Roberts W, Brian J, Szatmari P. Behavioral manifestations of autism in the first year of life. Int $\mathrm{J}$ Dev Neurosci 2005;23:143-152.

18. Chawarska K, Klin A, Paul R, Macari S, Volkmar F. A prospective study of toddlers with ASD: Short-term diagnostic and cognitive outcomes. J Child Psychol Psychiatry 2009;50:1235-1245.

19. CDC. Prevalence of autism spectrum disorders-Autism and Developmental Disabilities Monitoring Network, United States, 2006. MMWR Surveill Summ 2009;58:1-20.

20. Shattuck PT, Durkin M, Maenner M, et al. Timing of identification among children with an autism spectrum disorder: findings from a population-based surveillance study. J Am Acad Child Adolesc Psychiatry 2009;48:474-483.

21. Dawson G. Early behavioral intervention, brain plasticity, and the prevention of autism spectrum disorder. Dev Psychopathol 2008;20:775-803.

22. Buzsaki G. Rhythms of the brain. Oxford University Press, New York, NY, 2006.

23. Belmonte MK, Allen G, Beckel-Mitchener A, Boulanger LM, Carper RA, Webb SJ. Autism and abnormal development of brain connectivity. J Neurosci 2004;24:9228-9231.

24. Geschwind DH, Levitt P. Autism spectrum disorders: developmental disconnection syndromes. Curr Opin Neurobiol 2007;17:103111.

25. Wass S. Distortions and disconnections: disrupted brain connectivity in autism. Brain Cogn 2011;75:18-28.

26. Scott-Van Zeeland AA, Abrahams BS, Alvarez-Retuerto AI, et al. Altered functional connectivity in frontal lobe circuits is associated with variation in the autism risk gene CNTNAP2. Sci Trans1 Med 2010;2:56ra80.

27. Kilb W. Development of the GABAergic system from birth to adolescence. Neuroscientist 2012;18:613-630.

28. Betancur C, Sakurai T, Buxbaum JD. The emerging role of synaptic cell-adhesion pathways in the pathogenesis of autism spectrum disorders. Trends Neurosci 2009;32:402-412.

29. Ebert DH, Greenberg ME. Activity-dependent neuronal signalling and autism spectrum disorder. Nature 2013;493:327-337.

30. Bilimoria PM, Stevens B. Microglia function during brain development: New insights from animal models. Brain Res 2014;1-11.

31. Gogolla N, Leblanc JJ, Quast KB, Südhof TC, Fagiolini M, Hensch TK. Common circuit defect of excitatory-inhibitory balance in mouse models of autism. J Neurodev Disord 2009;1:172-181.

32. Blatt GJ, Fatemi SH. Alterations in GABAergic biomarkers in the autism brain: Research findings and clinical implications. Anat Rec 2011;294:1646-1652.

33. Lawrence YA, Kemper TL, Bauman ML, Blatt GJ. Parvalbumin-, calbindin-, and calretinin-immunoreactive hippocampal interneuron density in autism. Acta Neurol Scand 2010;121:99-108.

34. Nagarajan RP, Hogart AR, Gwye Y, Martin MR, LaSalle JM. Reduced $\mathrm{MeCP} 2$ expression is frequent in autism frontal cortex and correlates with aberrant MECP2 promoter methylation. Epigenetics 2006;1:37-41.

35. Chao H-T, Chen H, Samaco RC, et al. Dysfunction in GABA signalling mediates autism-like stereotypies and Rett syndrome phenotypes. Nature 2010;468:263-269.

36. Pizzarelli R, Cherubini E. Alterations of GABAergic signaling in autism spectrum disorders. Neural Plast 2011;2011:297153.

37. Wang L, Berry-Kravis E, Hagerman R. Fragile X: leading the way for targeted treatments in autism. Neurotherapeutics 2010;7:264274. 
38. Selby L, Zhang C, Sun QQ. Major defects in neocortical GABAergic inhibitory circuits in mice lacking the fragile $\mathrm{X}$ mental retardation protein. Neurosci Lett 2007;412:227-232.

39. Curia G, Papouin T, Séguéla P, Avoli M. Downregulation of tonic GABAergic inhibition in a mouse model of fragile $\mathrm{X}$ syndrome. Cereb Cortex 2009;19:1515-1520.

40. Centonze D, Rossi S, Mercaldo V, et al. Abnormal striatal GABA transmission in the mouse model for the fragile $\mathrm{X}$ syndrome. Biol Psychiatry 2008;63:963-973.

41. Olmos-Serrano JL, Paluszkiewicz SM, Martin BS, Kaufmann WE, Corbin JG, Huntsman MM. Defective GABAergic neurotransmission and pharmacological rescue of neuronal hyperexcitability in the amygdala in a mouse model of fragile $\mathrm{X}$ syndrome. J Neurosci 2010;30:9929-9938.

42. Tabuchi K, Blundell J, Etherton MR, et al. A neuroligin-3 mutation implicated in autism increases inhibitory synaptic transmission in mice. Science 2007;318:71-76.

43. Toro R, Konyukh M, Delorme R, et al. Key role for gene dosage and synaptic homeostasis in autism spectrum disorders. Trends Genet 2010;26:363-372.

44. Voineagu I, Wang X, Johnston P, et al. Transcriptomic analysis of autistic brain reveals convergent molecular pathology. Nature 2011;474:380-384.

45. Wills S, Rossi CC, Bennett J, et al. Further characterization of autoantibodies to GABAergic neurons in the central nervous system produced by a subset of children with autism. Mol Autism 2011;2:1-14.

46. Schafer DP, Lehrman EK, Kautzman AG, et al. Microglia sculpt postnatal neural circuits in an activity and complement-dependent manner. Neuron 2012;74:691-705.

47. Kim YS, Harry GJ, Kang HS, et al. Altered cerebellar development in nuclear receptor TAK1/TR4 null mice is associated with deficits in GLAST+ Glia, alterations in social behavior, motor learning, startle reactivity, and microglia. Cerebellum 2010;9:310-323.

48. Auerbach BD, Osterweil EK, Bear MF. Mutations causing syndromic autism define an axis of synaptic pathophysiology. Nature 2011;480:63-68.

49. Flores CE, Mendez P. Shaping inhibition: Activity dependent structural plasticity of GABAergic synapses. Front Cell Neurosci 2014;8:1-13.

50. Modahl C, Green LA, Fein D, et al. Plasma oxytocin levels in autistic children. Biol Psychiatry 1998;43:270-277.

51. Aoki Y, Yahata N, Watanabe T, et al. Oxytocin improves behavioural and neural deficits in inferring others' social emotions in autism. Brain 2014;137:3073-3086.

52. Guastella AJ, Gray KM, Rinehart NJ, et al. The effects of a course of intranasal oxytocin on social behaviors in youth diagnosed with autism spectrum disorders: a randomized controlled trial. J Child Psychol Psychiatry 2014 Aug 2 [Epub ahead of print].

53. Owen SF, Tuncdemir SN, Bader PL, Tirko NN, Fishell G, Tsien RW. Oxytocin enhances hippocampal spike transmission by modulating fast-spiking interneurons. Nature 2013;500:458-462.

54. Port RG, Gandal MJ, Roberts TPL, Siegel SJ, Carlson GC. Convergence of circuit dysfunction in ASD: A common bridge between diverse genetic and environmental risk factors and common clinical electrophysiology. Front Cell Neurosci 2014;8:1-14.

55. Gandal MJ, Edgar JC, Ehrlichman RS, Mehta M, Roberts TPL, Siegel SJ. Validating $\gamma$ oscillations and delayed auditory responses as translational biomarkers of autism. Biol Psychiatry 2010;68: $1100-1106$.

56. Edgar JC, Khan SY, Blaskey L, et al. Neuromagnetic oscillations predict evoked-response latency delays and core language deficits in autism spectrum disorders. J Autism Dev Disord 2015;45:395-405.

57. Wilson TW, Rojas DC, Reite ML, Teale PD, Rogers SJ. Children and adolescents with autism exhibit reduced MEG steady-state gamma responses. Biol Psychiatry 2007;62:192-197.

58. Milne E. Increased intra-participant variability in children with autistic spectrum disorders: evidence from single-trial analysis of evoked EEG. Front Psychol 2011;2:51.

59. Dinstein I, Heeger DJ, Lorenzi L, Minshew NJ, Malach R, Behrmann M. Unreliable evoked responses in autism. Neuron 2012;75:981-991.

60. Haigh SM, Heeger DJ, Dinstein I, Minshew N, Behrmann M. Cortical variability in the sensory-evoked response in autism. J Autism Dev Disord 2014 Oct 19 [Epub ahead of print].

61. Dinstein I, Thomas C, Humphreys K, Minshew N, Behrmann M, Heeger DJ. Normal movement-selectivity in autism. Neuron 2011;66:461-469.

62. Khan S, Gramfort A, Shetty NR, et al. Local and long-range functional connectivity is reduced in concert in autism spectrum disorders. Proc Natl Acad Sci U S A 2013;110:3107-3112.

63. Wulff P, Ponomarenko AA, Bartos M, et al. Hippocampal theta rhythm and its coupling with gamma oscillations require fast inhibition onto parvalbumin-positive interneurons. Proc Natl Acad Sci U S A 2009;106:3561-3566.

64. Freeman WJ, Rogers LJ, Walter J. Fine temporal resolution of analytic phase reveals episodic synchronization by state transitions in gamma EEGs. J Neurophysiol 2002;87:937-945.

65. Thatcher RW, North DM, Neubrander J, Biver CJ, Cutler S, Defina P. Autism and EEG phase reset: Deficient GABA mediated inhibition in thalamo-cortical circuits. Dev Neuropsychol 2009;34:780800 .

66. Nelson CA, McCleery JP. Use of event-related potentials in the study of typical and atypical development. J Am Acad Child Adolesc Psychiatry 2008;47:1252-1261. 